\title{
The ability to successfully include primary dental care practitioners in commissioning groups
}

\author{
N. M. Jessop, ' E. J. Kay, ${ }^{2}$ A. C. Mellor, ${ }^{3}$ J. G. Whittle, ${ }^{4}$ and A. Jenner, ${ }^{5}$
}

\begin{abstract}
Objectives To evaluate two total purchasing (TP) sites and ascertain whether general dental practitioners (GDPs) could be successfully included in the total purchasing model of health care commissioning. Another objective was to examine the role GDPs may play in future primary care commissioning groups and trusts.

Design An observational cross-sectional study of two dental purchasing pilots in the North West Region.

Subjects Two TP sites were studied, one in South Cheshire, the other East Lancashire. Each TP organisation had a dental subgroup which had a formal structure and was given an active role in dental healthcare service decision making.

Main outcome measures General dental practitioners' ability to manage dental health care provision in the selected sites and the suitability and effectiveness of the services managed by them. Results GDPs were willing and able to form cohesive primary care commissioning organisations but attitudes in secondary care prevented the implementation of their decisions.

Conclusions Structural, organisational and psychological changes would be necessary if GDP led purchasing were to be successfully implemented. Such changes are vital if GDPs and the dental profession are to have an effective role in the newly formed primary care groups and trusts.
\end{abstract}

\footnotetext{
Two dental total purchasing pilots were established in the 1 North West of England in 1996 and an evaluation was carried out to assess whether general dental practitioners (GDPs) could be successfully incorporated in the total purchasing (TP) model of primary care led commissioning. General medical practitioners (GMPs) have been established as the principal primary care decision makers in commissioning models such as fundholding, total purchasing and, subsequent to this study, in primary care groups and trusts. This has given them experience in the management of healthcare services and of working together in 'coherent' organisational structures. In contrast, at the time of this study, primary care dental practitioners had traditionally been detached from colleagues and almost completely excluded from service decisions

${ }^{1}$ Post Doctoral Research Fellow, Department of Oral Health and Development,

$2^{*}$ Professor of Dental Health Service Research, ${ }^{3}$ Senior Lecturer in in Primary

Dental Care, University Dental Hospital of Manchester, Higher Cambridge

Street, Manchester M16 6FH, ${ }^{4}$ Consultant in Dental Public Health, East

Lancashire Health Authority, 31/33 Kenyon Road, Lomeshaye Industrial Estate,

Nelson BB9 5SZ, ${ }^{5}$ Consultant in Dental Public Health, South Cheshire Health

Authority, 1829 Building, Countess of Chester Health Park, Liverpool Road,

Chester $\mathrm{CH} 21 \mathrm{BQ}$

${ }^{*}$ Correspondence to: $E$. Kay

email:E.Kay@man.ac.uk

REFEREED PAPER

Received 20.10.99; Accepted 03.04.00

(c) British Dental Journal 2000; 189: 675-677
}

about secondary dental care provision. The total purchasing pilots described in this paper offered primary care dental professionals the opportunity to take an active role in decisions about the oral healthcare services offered to their patients.

The aims and objectives of the dental total purchasing sites A key aim of the research programme was to assess the ability and willingness of GDPs to participate in primary care led commissioning organisations. The key assessment criteria were that dental practitioners had to be capable of cohesive decision-making and able to maintain motivation. If these requirements were achieved, the research aimed to evaluate the service decisions made by examining the suitability of the new services for the populations that the GDPs represented.

\section{The dental total purchasing sites}

Two total purchasing sites were selected for the dental pilot studies as GDPs in each locality had expressed an interest in taking part. The selected TP sites had differing sociodemographic structures and levels of oral health.

The organisational structure of the dental TP groups and their relationship with participating medical practitioners were examined qualitatively. This approach enabled an assessment of whether GDPs could work together, and with medical colleagues, in a primary care organisation, which required co-operation between and among all participants.

The ability of GDPs to form consensus decisions on dental health care provision was also studied. The decisions made by them were examined in relation to their suitability for the population the GDPs represented. The research question was, therefore: 'Are primary dental practitioners the best people to determine the oral health services offered to their registered population?'

The final aspect studied in the research programme was the ability of GDPs to implement their proposed changes and whether motivation was maintained throughout the course of the pilot projects.

\section{Establishment of organisational structure}

Dental total purchasing sub-groups were successfully established in both pilot sites. The managerial structure of the dental sub-groups was as follows:

- A lead GDP to advise and act as a contact for other practitioners in the group.

- A Consultant in Dental Public Health to advise on purchasing decisions and ensure that any proposed changes to services adhered to the Health Authority's overall strategy.

- A lead GMP to form a link between the medical and dental TP groups.

- The TP project manager to help with contracting and other health management issues. 
Evening meetings were held for dental practitioners in each site. The concept of total purchasing was discussed and potential beneficial service changes considered. These voluntarily attended meetings were well supported by GDPs and also attracted GMP participation. The GMPs offered advice to the dental project as they had the advantage of commencing their pilot project a year before the establishment of the dental pilot.

\section{Decisions about local dental service provision}

In both sites, service decisions were outlined and agreed by GDPs at an early stage of the pilots' development. Each dental purchasing group believed that a principal improvement to local dental care provision would be the setting up of 'outreach' clinics. These clinics were to be staffed by secondary care clinicians but would be based as close to the population using them as feasible, possibly in local 'cottage hospitals'.

This consensus of opinion by GDPs in each site showed they were able to make cohesive group decisions on service provision. They also agreed referral protocols. When the project commenced, data showed that there were some key hospitals providing secondary dental care to the populations of each locality. Practitioners were however willing to change their referral habits in order to purchase an outreach clinic for their patients. They saw such a development as offering a service with increased accessibility and convenience. The dentists also felt that the outreach clinics would offer an opportunity to improve communication between primary and secondary care providers. The decision to try to purchase outreach services was unanimous among the participating dental practitioners.

To provide this new service, funds had to be identified from the total purchasing budget. This process, like the decision on service provision, needed a cohesive approach from the participating GDPs. In both pilot sites, a methodology was devised to access the funds necessary to purchase an outreach service.

\section{The approach to achieve cost savings}

The costs of some service provision had to be reduced in order to have resources to purchase outreach services. The approach adopted by the GDPs was to reduce the purchasing of unnecessary secondary care activity. This aim was achieved using the following measures:

- Patients were only referred if they were identified as being treatable in a cost effective manner.

- Whenever appropriate, patients would be referred to primary care specialists.

- Colleagues would refer within the same practice wherever possible.

\section{The failure to purchase outreach services}

In both sites, each total purchasing group failed to gain outreach services for their patients. Neither TP group could successfully identify and redirect resources from their budget in a way which was acceptable to their respective health authorities. However, in East Lancashire cost savings were made in the dental total purchasing budget which had been provided by the North West Regional Office (NWRO) of the Department of Health. It was agreed by NWRO and East Lancashire Health Authority that this funding could be directed towards purchasing an outreach service.

After consideration and discussion with the principal local hospital provider, it was decided by the total purchasing GDPs that they should purchase an orthodontic outreach service. This purchasing decision was made, based on the potential benefit to the population and the practicalities of establishing an outreach service. The perceived potential benefits of an orthodontic outreach clinic based close to the local community were as follows:

- A reduction in the time children are absent from school.
- A reduction in the inconvenience and possible absence from work for parents/guardians.

- A reduction in treatment 'drop-out' rates due to the above inconveniences (in 1996 a report indicated that $20 \%$ of orthodontic treatment in the North West region was not completed). ${ }^{1}$

- Improved communication and professional understanding between GDPs and orthodontic clinicians.

A dental suite in the local cottage hospital was available to the total purchasing group. It was already used for orthodontic clinics by the local community dental service. An orthodontic clinic also required significantly less capital expenditure than, for example, an oral surgery service, which would need expensive apparatus, such as resuscitation facilities etc.

Although funding was identified which would meet the costs calculated by the local hospital provider, an outreach clinic was not established. This was entirely caused by reluctance by secondary care clinicians to staff such a service.

\section{Maintenance of motivation}

Motivation was high among GDPs during the start of the projects, as shown by attendance at voluntary meetings. Active participation in the pilot project declined in the East Lancashire site after continual failure to implement service change. The site in South Cheshire experienced a similar decline in motivation. However, the successful bid to join the Personal Dental Services pilot projects (established recently to facilitate a more responsive dental service) renewed the original enthusiasm of GDPs.

\section{The lessons from the dental total purchasing pilots Group cohesion}

Effective participation in total purchasing required a fundamental change in the organisation of local dental services and a transition of attitude in those who provided them. Despite the isolation of GDPs in the past, they were found to be capable and willing to take part in a primary care centred decision-making organisation. In fact, perceived neglect of their opinions in previous decision-making seemed to increase their desire to become actively involved in an organisation that empowered them to shape the local provision of service.

Unlike the medical practitioners in each of the TP sites, GDPs had not had any previous experience of NHS purchasing. The fundholding system had allowed GMPs to buy healthcare services for their practice population, so they were well aware of the purchasing process. However, some GMPs had decided to buy additional services for patients within their own medical practices. The decision by policy makers to create an 'equitable' NHS made these extra services, which were available only in fundholding practices, untenable. Yet, medical practices remained reluctant to remove these additional services from their practice population and to an extent this reduced the cohesion of the medical group. In contrast, the dental TP pilots presented a new opportunity to GDPs to actively direct policy about hospital dental care provision. Potentially, such changes would release more resources to the primary care setting. As such, the response to TP was positive and potential changes in service provision were agreed.

Total purchasing medical colleagues in each site were highly cooperative with the dental group. The medical practitioners helped in negotiations with secondary care providers and were willing to try to help the dental project improve oral health in each area. The following proposals are examples of initiatives supported by the GMPs:

- Increasing dental registration of children under 5 years through encouragement by GMPs and their support staff, eg health visitors.

- Increasing dental registration of adults over 65 years through 
encouragement by GMPs and their support staff (an effort towards early detection of oral cancer).

- Making available 'sugar-free' medicines to children.

- The inclusion of dental pain in a minor ailments booklet which aimed to reduce emergency calls to doctors. Dental pain had been omitted from the draft booklet prior to dental participation in the TP scheme.

Active collaboration between the medical and dental TP pilots was observed. Positive achievements by either professional group supported the concept of primary care purchasing as a whole. The need to collaborate in order to strengthen the voice of primary care in decision-making was clearly recognised by all concerned.

\section{The dental practitioner decision-making process}

Given the differing sociodemographic structures and the oral health needs of the two dental TP pilot areas, the similarity in proposed service development was somewhat unexpected. This could be taken to indicate that the total purchasing of dental services was not specifically responsive to the needs of the local population but simply allowed a general service requirement to be brought about by the practitioners. If the purchasing specifications decided upon were appropriate in both sites, then this perhaps indicates that there is a requirement across the country for outreach clinics if efficient and effective delivery of care is to be achieved. If this is the case, extensive dental involvement in TP and other primary care led commissioning organisations may be unnecessary. If they are universally needed, the increased use of outreach clinics could be enacted centrally, at less expense, as dental purchasing bodies are not needed to influence decisions which apply to all communities. However, this is highly speculative, as the processes in each site when discussing potential improvements to service were quite different. In the site located in South Cheshire, GDPs had strong views on what should be changed in the delivery of dental care, particularly in relation to secondary providers. This was the case even though data were not initially available to support their views. In the site located in East Lancashire, opinion was more conservative and a consensus of opinion regarding operational change was only arrived at after careful analysis of the current position.

\section{The primary and secondary care relationship}

The fact that dentistry had not previously been involved in the fundholding system had some advantages when the GDPs became total purchasers; it also acted as a key disadvantage. Unlike hospital medical clinicians, secondary care dental clinicians were not used to negotiating service provision with primary care practitioners. This resulted in reluctance among hospital clinicians to accept and act upon primary care led initiatives.

This attitude was evident in both TP sites. In one site, the total purchasing GDPs identified funds to support the provision of a new and, in their view, much needed service. Prolonged negotiation took place between TP representatives, hospital management and secondary care clinicians. The hospital provider calculated a price for the service and the TP dental sub-group agreed to purchase the service for the requested amount. Yet, the service was not implemented. This situation occurred because secondary care clinicians were unwilling to staff the clinic, as they believed this would prove detrimental to hospital services. An attempt to staff the clinic, with a senior registrar wwho would perform treatments after a consultant opinion had been given, also failed because staff were unwilling to provide treatment in an 'outreach' setting.

Lack of available hospital referral activity data prevented the TP site in South Cheshire from commissioning any new services. In East Lancashire, the dental TP sub-group identified funds to purchase a new service for their practice population. A cohesive service decision had been achieved and dental practitioners shown that they could be 'total purchasers'. Obstruction by a dominant hospital provider, rather than GDP inability to purchase, prevented them from carrying out their proposed changes to service.

\section{Motivation}

Motivation was initially high in both sites, but declined because of lack of progress. A primary care organisation which relies upon professionals' voluntary participation needs to make progress, or support will decline. The research programme proved that primary dental care practitioners wanted to help shape local dental care provision. This was further demonstrated when the locality in South Cheshire successfully joined the newly introduced Personal Dental Services pilots. This resulted in a renewed vigour among total purchasing GDPs who seized upon this fuller opportunity to shape dental health care provision to their local population. However, without visible results, such enthusiasm will inevitably decline.

\section{Conclusion}

The failure of both sites to use total purchasing to change local dental healthcare delivery was caused by the hospital providers' unwillingness to accept change, rather than any shortcoming among the primary care purchasers. If the attitudes of secondary care providers are not changed, there is a real risk that dental services will become a low priority for the new Primary Care Groups (PCGs) and Primary Care Trusts (PCTs) and, based on the evidence presented here, it would seem that Oral Health Advisory Groups (OHAGs) will not be able to shape service decisions to any substantial degree. Should secondary dental care providers continue to resist change, it is probable that GDPs will lose interest in health service development. Although the OHAGs will represent the dental profession's views to PCG/T committees, demotivation among primary care practitioners and consequently reduced influence is a real danger. Dental representation on PCG/T committees may therefore become little more than 'lip-service' to the profession. This will inevitably be to the detriment of dental service provision and should be avoided. If GMPs are thought to be the people best placed to commission secondary medical services, then why are GDPs not considered to be best placed to commission secondary dental services? The answers to this question lie in part in secondary care management information systems. These will be discussed in the next paper in this series.

1. Taylor G, Jenner A. Orthodontic treatment for children in the North West Region-a guide for purchasers. North West Region Dental Public Health Resource Centre, July 1996. 